\title{
NOTE ON THE SPECTRAL REPRESENTATION OF A BOUNDED NORMAL MATRIX
}

\author{
EARL A. CODDINGTON
}

Every bounded normal (infinite) matrix $N$ which possesses a bounded reciprocal may be written as a product of its polar factors as $N=P U=U P$, where $P$ is positive definite and $U$ is unitary. Using the corresponding spectral representations for $P$ and $U$, a spectral representation of $N$ may be obtained by means of Stieltjes integrals. Also, the cartesian factorization $N=H_{1}+i H_{2}$, where $H_{1}$ and $H_{2}$ are Hermitian and commutable, together with the spectral representations of $H_{1}$ and $H_{2}$ may be employed to obtain a spectral representation of $N$ (cf. $[1,6]^{1}$ for references). It seems more natural to proceed directly by using the result on the moment problem for distribution functions in more than one dimension. The spectral representation of $N$ is a consequence of the theorem below, which is the two-dimensional analogue of one employed by M. H. Martin [2] in obtaining the spectral representation of a bounded Hermitian matrix. The theorem holds in general for any finite number of bounded Hermitian matrices which commute with each other, but we consider the case of two such matrices for simplicity.

Notation. Let $x, y$ denote one-column vectors with an infinity of (complex) components $x_{i}, y_{i}, i=1,2, \cdots$. An infinite matrix $A$ is said to be bounded if the least upper bound (l.u.b.) of the set of numbers $\left|y^{*} A x\right|$ is finite $\left(y^{*}=\bar{y}^{\prime}\right.$, the conjugate transpose of $y$ ), where $x$ and $y$ range independently over the unit Hilbert sphere, that is, $|x|=\left(\sum_{i=1}^{\infty}\left|x_{i}\right|^{2}\right)^{1 / 2}=1,|y|=\left(\sum_{i=1}^{\infty}\left|y_{i}\right|^{2}\right)^{1 / 2}=1$. The matrix $H$ is said to be Hermitian if $H=H^{*}$. For Hermitian matrices $x^{*} H x$ is real and

$$
\underset{|x|=|y|=1}{\text { l.u.b. }}\left|y^{*} H x\right|=\underset{|x|=1}{\operatorname{lu} . b .}\left|x^{*} H x\right| \text {. }
$$

If $H$ is Hermitian and the greatest lower bound (g.1.b.) of the set of numbers $x^{*} H x$ is non-negative, that is,

$$
\underset{|x|=1}{\text { g.l.b. }} x^{*} H x \geqq 0,
$$

then $H$ is said to be non-negative definite. A positive definite matrix, $P$, is a Hermitian matrix satisfying

Received by the editors October 6, 1947.

1 Numbers in brackets refer to references cited at the end of the paper. 


$$
\underset{|x|=1}{\text { g.l.b. }} x^{*} P x>0
$$

We denote the unit matrix by $E$. A unitary matrix, $U$, is one for which $U U^{*}=U^{*} U=E$. $N$ is said to be a normal matrix if $N N^{*}=N^{*} N$.

We first prove the following lemima.

LEMma. If $P_{1}$ and $P_{2}$ are two positive definite bounded matrices for which
(a) $P_{1} P_{2}=P_{2} P_{1}$,
(b) $\underset{|x|=1}{\operatorname{l.u.b} .} x^{*} P_{1} x<1, \underset{|x|=1}{\text { l.u.b. }} x^{*} P_{2} x<1$

then there exist four bounded Hermitian matrices $A_{1}, A_{2}, B_{1}, B_{2}$ such that

(i) $A_{1}^{2}=P_{1}, A_{2}^{2}=P_{2}, B_{1}^{2}=E-P_{1}, B_{2}^{2}=E-P_{2}$,

(ii) $A_{1}, A_{2}, B_{1}, B_{2}$ commute with each other.

Proof. Under the assumptions the existence of $A_{1}, A_{2}, B_{1}, B_{2}$ is guaranteed (cf. [3]). In fact, the "square roots" $A_{i}$ and $B_{i}$ may be represented as series

$$
A_{i}=\sum_{n=0}^{\infty} c_{n}\left(E-P_{i}\right)^{n}, \quad B_{i}=\sum_{n=0}^{\infty} c_{n} P_{i}^{n}, \quad i=1,2,
$$

where $c_{0} c_{0}=1, c_{0} c_{1}+c_{1} c_{0}=-1$,

$$
\sum_{m=0}^{n} c_{m} c_{n-m}=0, \quad n=2,3, \cdots \text {. }
$$

$A_{1}$ clearly commutes with $B_{1}$, and similarly for $A_{2}$ and $B_{2}$. By (a) $A_{1}$ and $A_{2}$ commute, as well as $B_{1}$ and $B_{2}$. Therefore $A_{1}, A_{2}, B_{1}, B_{2}$ all commute with each other.

TheOREM. If $H_{1}$ and $H_{2}$ are bounded Hermitian matrices which commute and

$$
\mu_{y}(m, n)=y^{*} H_{1}^{m} H_{2}^{n} y, \quad m, n=0,1,2, \cdots, H_{1}^{0}=H_{2}^{0}=E,
$$

where $y$ is a fixed vector for which $|y|=1$, then there exists an additive monotone set function $\phi=\phi_{y}=\phi_{y}(S)$ of the set $S$ in the $u$, v-plane $(\phi(S) \equiv 0$ if $S$ outside a certain bounded rectangle) such that

$$
\mu_{y}(m, n)=\int_{-\infty}^{+\infty} \int_{-\infty}^{+\infty} u^{m v^{n}} \phi_{y}\left(d S^{u v}\right), \quad m, n=0,1,2, \cdots .
$$

Proof. There exist real numbers $\alpha_{1}, \beta_{1}, \alpha_{2}, \beta_{2}$ for which

$$
P_{1}=\alpha_{1} H_{1}+\beta_{1} E, \quad P_{2}=\alpha_{2} H_{2}+\beta_{2} E \quad\left(\alpha_{1}, \alpha_{2}>0\right)
$$


satisfy the conditions of the lemma. Also, if a function $\phi$ exists for $P_{1}$ and $P_{2}$, one will exist for $H_{1}$ and $H_{2}$ since the transformation $\left(^{*}\right)$ only results in a translation and stretching of the spectrum of $\phi$. Therefore we may assume $H_{1}$ and $H_{2}$ have the same properties as $P_{1}$ and $P_{2}$ of the lemma.

For a given double sequence of numbers $\mu(m, n)$ a necessary and sufficient condition that an additive monotone set function $\phi(S)$ exist with moments

$$
\mu(m, n)=\int_{-\infty}^{+\infty} \int_{-\infty}^{+\infty} u^{m v^{n}} \phi\left(d S^{u v}\right), \quad m, n=0,1,2, \cdots
$$

is that

$$
\Delta_{1}^{k} \Delta_{2}^{h} \mu(m, n) \geqq 0, \quad k, h, m, n=0,1,2, \cdots
$$

where

$$
\Delta_{1}^{k} \Delta_{2}^{h} \mu(m, n)=\sum_{r=0}^{k} \sum_{s=0}^{h}\left(\begin{array}{l}
k \\
r
\end{array}\right)\left(\begin{array}{l}
h \\
s
\end{array}\right)(-1)^{r+s} \mu(m+r, n+s)
$$

(cf. [4] and [5]). For $\mu(m, n)=\mu_{y}(m, n)=y^{*} H_{1}^{m} H_{2}^{n} y$,

$$
\begin{aligned}
\Delta_{1}^{k} \Delta_{2}^{h} \mu_{y}(m, n) & =\sum_{r=0}^{k} \sum_{s=0}^{h}\left(\begin{array}{c}
k \\
r
\end{array}\right)\left(\begin{array}{l}
h \\
s
\end{array}\right)(-1)^{r+s}\left(y^{*} H_{1}^{m+r} H_{2}^{n+s} y\right) \\
& =y^{*}\left(\sum_{r=0}^{k} \sum_{s=0}^{h}\left(\begin{array}{c}
k \\
r
\end{array}\right)\left(\begin{array}{c}
h \\
s
\end{array}\right)(-1)^{r+s} H_{1}^{m+r} H_{2}^{n+s}\right) y \\
& =y^{*} H_{1}^{m} H_{2}^{n}\left(E-H_{1}\right)^{k}\left(E-H_{2}\right)^{h} y
\end{aligned}
$$

since $H_{1}$ and $H_{2}$ commute. Hence we must show that the matrix polynomial $H_{1}^{m} H_{2}^{n}\left(E-H_{1}\right)^{k}\left(E-H_{2}\right)^{h}$ is non-negative definite for $k, h, m, n$ $=0,1,2, \cdots$.

From the lemma we have the existence of $A_{1}, A_{2}, B_{1}, B_{2}$ and it follows that

$$
\begin{aligned}
H_{1}^{m} H_{2}^{n}\left(E-H_{1}\right)^{k}\left(E-H_{2}\right)^{h} & =\left(A_{1}^{2}\right)^{m}\left(A_{2}^{2}\right)^{n}\left(B_{1}^{2}\right)^{k}\left(B_{2}^{2}\right)^{h} \\
& =\left(A_{1}^{m} A_{2}^{n} B_{1}^{k} B_{2}^{h}\right)\left(A_{1}^{m} A_{2}^{n} B_{1}^{k} B_{2}^{h}\right) \\
& =\left(A_{1}^{m} A_{2}^{n} B_{1}^{k} B_{2}^{h}\right)\left(A_{1}^{m} A_{2}^{n} B_{1}^{k} B_{2}^{h}\right)^{*} .
\end{aligned}
$$

Since any bounded matrix of the form $C C^{*}$ is non-negative definite, the theorem is proved.

If $N$ is a normal bounded matrix, $N=H_{1}+i H_{2}$ where $H_{1}$ $=\left(N+N^{*}\right) / 2, H_{2}=\left(N-N^{*}\right) / 2 i$ and $H_{1}$ and $H_{2}$ are both bounded Hermitian matrices which commute with each other since $N$ is normal, 
that is, $H_{1}$ and $H_{2}$ satisfy the conditions of the theorem. Hence, we may write

$$
\begin{aligned}
\sum_{k=0}^{n}\left(\begin{array}{l}
n \\
k
\end{array}\right)(i)^{n-k}\left(y^{*} H_{1}^{k} H_{2}^{n-k} y\right) & \\
& =\sum_{k=0}^{n}\left(\begin{array}{l}
n \\
k
\end{array}\right)(i)^{n-k} \int_{-\infty}^{+\infty} \int_{-\infty}^{+\infty} u^{k} v^{n-k} \phi_{y}\left(d S^{u v}\right),
\end{aligned}
$$

or

$$
y^{*}\left(H_{1}+i H_{2}\right)^{n} y=\int_{-\infty}^{+\infty} \int_{-\infty}^{+\infty}(u+i v)^{n} \phi_{y}\left(d S^{u v}\right) ;
$$

but this is the same as

$$
y^{*} N^{n} y=\int w^{n} \phi_{y}\left(d S^{w}\right)
$$

where $w=u+i v$ and the integration is over the entire $w$-plane.

With proper normalizations $\phi_{y}(S)$ may be made unique.

\section{REFERENCES}

1. D. Hilbert, Gesammelte Abhandlungen, Berlin, 1935, vol. 3, p. 122 (article by E. Hellinger).

2. M. H. Martin, On infinite orthogonal matrices, Amer. J. Math. vol. 54 (1932) pp. 625-631.

3. E. Hellinger and $\mathrm{O}$. Toeplitz, Integralgleichungen und Gleichungen mit unendlichvielen Unbekannten, Encyklopädie der Mathematischen Wissenshaften II C 13, p. 1567 , note 522 a.

4. T. H. Hildebrandt and I. J. Schoenberg, On linear functional operations and the moment problem for a finite interval in one or several dimensions, Ann. of Math. (2) vol. 34 (1933) pp. 317-328.

5. E. K. Haviland, On the momentum problem for distribution functions in more than one dimension, II, Amer. J. Math. vol. 58 (1936) pp. 164-168.

6. B. v. Sz. Nagy, Spektraldarstellung linearer Transformationen des Hilbertschen Raumes, Ergebnisse der Mathematik und ihrer Grenzgebiete, vol. 5, no, 5, p. 26.

The Johns Hopkins University 